
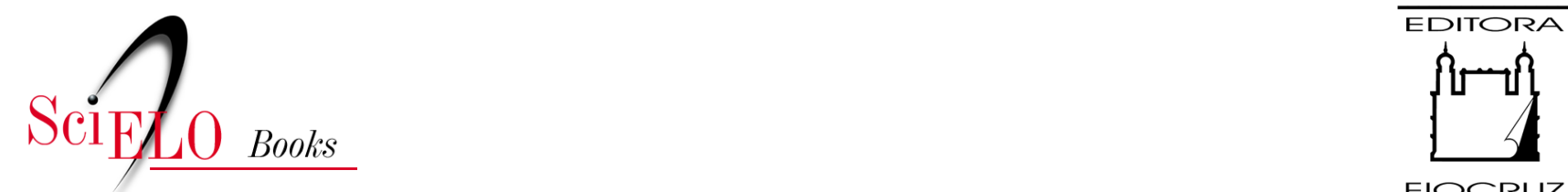

FIOCRUZ

\title{
III Anexos \\ Anexo G - Diretriz clínica para prevenção e tratamento do delirium em pacientes internados em serviços de pacientes em condições agudas e de cuidados gerais
}

\author{
Luciana Santos de Carvalho \\ Isabela Ribeiro Simões de Castro
}

\section{SciELO Books / SciELO Livros / SciELO Libros}

CARVALHO, L. S., and CASTRO, I. R. S. Diretriz clínica para prevenção e tratamento do delirium em pacientes internados em serviços de pacientes em condições agudas e de cuidados gerais. In: SOUSA, P., and MENDES, W., comps. Segurança do paciente: conhecendo os riscos nas organizações de saúde [online]. 2nd ed. rev. updt. Rio de Janeiro, RJ : CDEAD, ENSP, Editora FIOCRUZ, 2019, pp. 506509. ISBN 978-85-7541-641-9. https://doi.org/10.7476/9788575416419.0028. \section{International license.}

All the contents of this work, except where otherwise noted, is licensed under a Creative Commons Attribution 4.0

Todo o conteúdo deste trabalho, exceto quando houver ressalva, é publicado sob a licença Creative Commons Atribição 4.0. 


\section{Anexo G - Diretriz clínica para prevenção e tratamento do delirium em pacientes internados em serviços de pacientes em condições agudas e de cuidados gerais}

\section{Luciana Santos de Carvalho e Isabela Ribeiro Simões de Castro}

O delirium é um distúrbio neurocognitivo comum, marcado pela desatenção e falha da consciência, que se desenvolve em um curto período de tempo. Dois fatores de risco marcam a predisposição comum para o delirium: a idade avançada e a demência. Em geral, a prevalência de delirium em ambientes hospitalares varia de 18 a 35\%, de 26 a $62 \%$ em unidades de cuidados paliativos, e de 30 a $70 \%$ em cuidados intensivos, dependendo do perfil de pacientes e dos métodos de avaliação (Bush et al. 2017).

O objetivo da diretriz clínica para prevenção e tratamento do delirium é identificar pacientes com fatores de risco para delirium, diagnosticar e tratar prontamente as causas do delirium seguindo protocolos. O nível de evidência (Cochrane) dos resultados com o uso da diretriz é III. O tempo para implantação do protocolo é de um a dois anos. A dificuldade de implantação é média e depende de diversas áreas e da cultura existente na organização de saúde. Os impactos do uso da diretriz são:

* Redução de custo

腆 Redução de danos

滕 Redução de tempo de internação em unidades intensivas (medido nos últimos seis meses)

滕 Redução de readmissões (no período de 30 dias)

As consequências do delirium são déficits anatômicos, desequilíbrio (em razão de alterações nos níveis de serotonina, acetilcolina e deficiência de dopamina). Essas alterações podem ser secundárias a: redução no metabolismo cerebral; doença intracraniana primária; doenças sistémicas; infecção secundária do cérebro; agentes tóxicos exógenos; retirada de drogas e substâncias de uso crônico, tais como álcool ou agentes sedativo-hipnóticos; hipoxemia; distúrbios metabólicos; e a administração de medicamentos psicoativos, como os benzodiazepínicos. 


\section{Itens relevantes das diretrizes clínicas do Critical Care Medicine sobre delirium}

\section{As ações estratégicas são:}

滕 Avaliar o risco de todos os pacientes internados em unidades agudas e de cuidados gerais.

Reconhecer sinais e sintomas precocemente.

Tratar farmacologicamente o delirium.

\section{As ações mais operacionais são:}

䠊 Identificar pacientes com fatores de risco:

- variáveis não controláveis: idade e gravidade da doença existente (pré-existência de demência);

- variáveis controláveis: coma, uso de benzodiazepínicos, histórico de alcoolismo.

Manter familiares e cuidadores próximos o maior tempo possível.

路 Restituir óculos e aparelho auditivo o mais cedo possível.

Manter ambiente silencioso e com janelas para percepção de luz do dia.

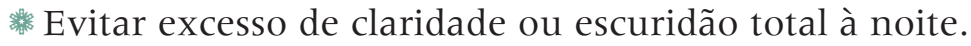

滕 Manter relógios, calendários e itens pessoais.

滕 Não acordar à noite para administrar medicação ou aferir sinais vitais, a menos que seja estritamente necessário.

Não interromper medicamentos psicoativos de uso habitual.

滕 Proporcionar analgesia e hidratação adequada no pós-operatório.

* Evitar contenção no leito/cama, a não ser quando for estritamente necessário para preservar a segurança do paciente.

滕 Mobilizar o paciente precocemente no leito/cama.

滕 Utilizar, preferencialmente, as ferramentas validadas para monitoramento do delirium em pacientes internados em unidades intensivas e clínicas como: The Confusion Assessment Method for the ICU - CAM-ICU ou The Intensive Care Delirium Screening Checklist - ICDSC.

Familiarizar o paciente com o ambiente, reforçando as orientações de uso de óculos e aparelhos auditivos, conforme necessário.
Resultados de estudos demonstram que altos níveis de ruídos, incluindo aqueles oriundos dos alarmes, não apenas interferem no cuidado, mas possuem, também, efeitos deletérios para a recuperação dos pacientes internados em terapia intensiva e na saúde dos profissionais que atuam nesses setores (Sampaio Neto et al. 2010). 


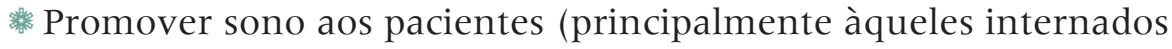
em unidades intensivas).

Monitorar a retirada do ventilador avaliando nível de consciência, nos casos de pacientes internados em unidades intensivas.

\section{As ações farmacológicas:}

* Evitar o uso de benzodiazepínicos em pacientes em risco de delirium, exceto se houver suspeita de abstinência desse tipo de droga.

* Não usar rivastigmina para reduzir a duração do delirium.

滕 Não utilizar haloperidol ou psicóticos atípicos para prevenir delirium.

橉 Evitar o uso de antipsicóticos em pacientes com prolongamento do segmento QT, história de Torsades de Pointes (taquicardia ventricular caracterizada por flutuação dos complexos QRS, tipicamente causada por um intervalo QT prolongado e podendo estar associada à administração de antipsicóticos) e em pacientes que recebem medicações para o prolongamento de segmento QT.

Usar dexmedetomidina em vez de infusões de benzodiazepínicos, quando a sedação for necessária em pacientes críticos com delirium, a menos que esteja relacionado ao álcool ou à retirada de benzodiazepínicos.

*studos recentes apontam efeitos positivos da melatonina na prevenção do delirium em pacientes idosos. Entretanto, a evidência científica ainda é considerada insuficiente para recomendar o seu uso para redução da incidência do delirium em pacientes internados, com 65 anos ou mais (CADTH 2016).

\section{Indicadores utilizados para monitorar a prevenção e tratamento do delirium em pacientes internados em unidades agudas e de cuidados gerais:}

滕 Percentual de pacientes com risco de desenvolver delirium.

橉 Percentual de pacientes monitorados diariamente para delirium.

路 Percentual de pacientes com delirium conduzidos por protocolos. 


\section{Referências}

Agency for Healthcare Research and Quality. Making health care safer: a critical analysis of patient safety practices. Rockville (MD): AHRQ; 2001 [citado 2014 ago 25]. Disponível em: ttp://www.ahrq. gov/research/findings/evidence-based-reports/ptsafetyuptp.html.

Agostini JV, Baker DI, Inouye SK, Bogardus Jr, ST. Prevention of delirium in older hospitalized patients. In: Agency for Healthcare Research and Quality. Making health care safer: a critical analysis of patient safety practices. Rockville (MD): AHRQ; 2001 [citado 2014 ago 25]. Chapter 28. Disponivel em: http://archive.ahrq.gov/clinic/ptsafety/chap28.htm

Barr J, Fraser GL, Puntillo K. Clinical practice guidelines for the management of pain, agitation, and delirium in adult patients in the intensive care unit. Crit Care Med. 2013 Jan;41(1):263-306.

Bush $\mathrm{SH}$, Marchington $\mathrm{KL}$, Agar M, et al. Quality of clinical practice guidelines in delirium: a systematic appraisal. BMJ Open 2017;7:e013809. doi: 10.1136/bmjopen-2016-013809.

Canadian Agency for Drugs and Technologies in Health. Sleep medications for the treatment or prevention of delirium: clinical effectiveness and guidelines. Ottawa: CADTH; 2016 Dec 9 [citado 2018 out 20]. Rapid response report. Disponível em: https://www.cadth.ca/sites/default/files/pdf/ htis/2016/RB1046\%20Sleep\%20Medications\%20for\%20Delirium\%20Final.pdf

Centers for Disease Control and Prevention. Medication Safety Program. Atlanta: CDC; 2014 [citado 2014 ago 25]. Disponível em: http://www.cdc.gov/medicationsafety/

Joint Commission. Advancing effective communication, cultural competence, and patientcentered care: a roadmap for the hospital. Oakbrook Terrace (IL): Joint Commission, 2010 [citado 2014 ago 25]. Disponível em: http://www.jointcommission.org/assets/1/6/ ARoadmapforHospitalsfinalversion727.pdf

National Priorities Partnership. Palliative care and end-of-life care. Washington, DC: National Quality Forum; 2010 [citado 2014 ago 25]. Disponível em: www.qualityforum.org/Publications/2010/11/ Palliative_Care_and_End-of-Life_Care_Convening_Meeting.aspx

Hughes RG, editor. Patient safety and quality: an evidence-based handbook for nurses. Rockville (MD): Agency for Healthcare Research and Quality; 2008 Apr.

Kleinpell RM, Fletcher K, Jennings BM. Reducing functional decline in the hospitalized elderly. In: Hughes RG, editor. Patient safety and quality: an evidence-based handbook for nurses. Rockville (MD): Agency for Healthcare Research and Quality; 2008 Apr [citado 2014 ago 25]. Disponível em: http://www.ahrq.gov/professionals/clinicians- providers/resources/nursing/resources/nurseshdbk/ KleinpellR_RFDHE.pdf

Vanderbilt University, Center for Health Services Research. ICU Delirium and Cognitive Impairment Study Group: training manual and instructional video. Nashville, TN: VUMC; 2013 [citado 2014 ago 25]. Disponível em: http://www.icudelirium.org/delirium/monitoring.html

Yale University. The Hospital Elder Life Program (HELP). New Haven, CT: School of Medicine; 2000 [citado 2014 ago 25]. Disponível em: http://www.hospitalelderlifeprogram.org/public/patientfamily.php 